PROCEEDINGS OF THE

AMERICAN MATHEMATICAL SOCIETY

Volume 127, Number 9, Pages 2505-2509

S 0002-9939(99)05275-2

Article electronically published on April 28, 1999

\title{
SOME COROLLARIES OF FROBENIUS' NORMAL $p$-COMPLEMENT THEOREM
}

\author{
YAKOV BERKOVICH
}

(Communicated by Ronald M. Solomon)

\begin{abstract}
For a prime divisor $q$ of the order of a finite group $G$, we present the set of $q$-subgroups generating $\mathrm{O}^{q, q^{\prime}}(G)$. In particular, we present the set of primary subgroups of $G$ generating the last member of the lower central series of $G$. The proof is based on the Frobenius Normal $p$-Complement Theorem and basic properties of minimal nonnilpotent groups. Let $G$ be a group and $\Theta$ a group-theoretic property inherited by subgroups and epimorphic images such that all minimal non- $\Theta$-subgroups ( $=\Theta_{1}$-subgroups) of $G$ are not nilpotent. Then (see the lemma), if $K$ is generated by all $\Theta_{1}$-subgroups of $G$ it follows that $G / K$ is a $\Theta$-group.
\end{abstract}

Let $p, q$ be distinct primes, $\pi(G)$ the set of all prime divisors of the order of a finite group $G$ (we consider only finite groups), $\pi$ a set of primes, $\operatorname{Syl}_{p}(G)$ the set of Sylow $p$-subgroups of $G, \Phi(G), \mathrm{F}(G), G^{\prime}$ and $\mathrm{Z}(G)$ the Frattini subgroup, the Fitting subgroup, the commutator subgroup and the center of $G$, respectively. In what follows, $\Theta$ is a nonempty group-theoretic property inherited by subgroups and epimorphic images and such that there exists a non- $\Theta$-group.

A group is said to be minimal nonnilpotent if it is not nilpotent but all its proper subgroups are nilpotent. Let $G$ be minimal nonnilpotent. It is known (see [Hup], Satz 3.5.2 - this theorem is due to O. Yu. Schmidt [S] and Yu. A. Golfand [Gol]; L. Redei $[\mathrm{R}]$ gave the complete classification of such groups) that

(i) $\pi(G)=\{p, q\}$ and $G=P Q$, where $P \in \operatorname{Syl}_{p}(G), G^{\prime}=Q \in \operatorname{Syl}_{q}(G)$;

(ii) $P$ is cyclic and $|P: P \cap \mathrm{Z}(G)|=p$;

(iii) $Q / Q \cap \mathrm{Z}(G)$ is a minimal normal subgroup of $G / Q \cap \mathrm{Z}(G), Q$ is special. (Recall that a $q$-group $Q$ is special if it is elementary abelian or $Q^{\prime}=\mathrm{Z}(Q)=\Phi(Q)$; in particular, the exponent of $Q$ is at most $q^{2}$.) It is known that if $q>2$, then $\exp (Q)=q$.

It is easy to check that a group possessing properties (i-iii) is minimal nonnilpotent. We call a group with this structure an $\mathrm{S}(p, q)$-group.

A group $G$ is said to be $p$-nilpotent if it has a normal $p$-complement. By Frobenius' Normal $p$-Complement Theorem and Ito's remark (see $[\mathrm{H}]$, Theorem 14.4.7, [Hup], Satz 4.5.4, or [I]) $G$ is $q$-nilpotent if and only if it has no S( $p, q)$-subgroups. A group $G$ is said to be $p$-closed if its Sylow $p$-subgroup is normal.

Received by the editors May 14, 1997.

1991 Mathematics Subject Classification. Primary 20D20.

Key words and phrases. Special $p$-group, minimal nonnilpotent (nonabelian, noncyclic, nonsolvable) group, $p$-nilpotent group, $p$-closed group, $\mathrm{S}(p, q)$-group, $\mathrm{B}(p, q)$-group.

The author was supported in part by the Ministry of Absorption of Israel.

(C)1999 American Mathematical Society 
Let $\mathrm{O}^{p}(G)$ be the subgroup generated by all $p^{\prime}$-elements of $G$, and $\mathrm{O}^{p, p^{\prime}}(G)$ the subgroup generated by all $p$-elements of $\mathrm{O}^{p}(G)$. Obviously, $G / \mathrm{O}^{p, p^{\prime}}(G)$ is $p$ nilpotent but, for every normal subgroup $N$ of $G$ properly contained in $\mathrm{O}^{p, p^{\prime}}(G)$, $G / N$ is not $p$-nilpotent. Let $\mathrm{H}(G)=\bigcap_{p \in \pi(G)} \mathrm{O}^{p}(G) ; \mathrm{H}(G)$ is the last member of the lower central series of $G$. It is clear that $\mathrm{O}^{p, p^{\prime}}(G) \leq \mathrm{H}(G)$.

Suppose that $\Phi(G) \leq N \unlhd G$. If $N / \Phi(G)$ has a normal $\pi$-Hall subgroup $K_{1} / \Phi(G)$, then $N$ has a normal $\pi$-Hall subgroup as well. Indeed, $K_{1}$ has a $\pi$-Hall subgroup $K$ and all such subgroups are conjugate in $K_{1}$, by the Schur-Zassenhaus Theorem. Therefore, $G=K_{1} \mathrm{~N}_{G}(K)=K \Phi(G) \mathrm{N}_{G}(K)=\mathrm{N}_{G}(K)$, and our claim follows since $K$ is a $\pi$-Hall subgroup of $N$. It follows from what has just been proved and the Schur-Zassenhaus Theorem that $\pi(G / \Phi(G))=\pi(G)$.

Let $G$ be an arbitrary group.

Definition 1. A group $G$ is said to be a $\mathrm{B}(p, q)$-group if $G / \Phi(G)$ is an $\mathrm{S}(p, q)$-group for some primes $p, q$.

Definition 2. A group $G$ is said to be a $\Theta_{1}$-group (= minimal non- $\Theta$-group) if it is not a $\Theta$-group but all its proper subgroups are $\Theta$-groups.

It is clear that $G$ is a $\Theta$-group if and only if it has no $\Theta_{1}$-subgroups.

It follows from the remark preceding Definition 1 that a $\mathrm{B}(p, q)$-group $G$ has the form $P Q$, where $P \in \operatorname{Syl}_{p}(G), G^{\prime}=Q \in \operatorname{Syl}_{q}(G),|P: P \cap \mathrm{Z}(G)|=p, P$ is cyclic and $Q / \Phi(Q)$ is a minimal normal subgroup of $G / \Phi(Q)$. It is clear that a nonnilpotent epimorphic image of a $\mathrm{B}(p, q)$-group is also a $\mathrm{B}(p, q)$-group. Let $Q>\{1\}$ be a $q$-group. There exists a $\mathrm{B}(p, q)$-group with Sylow subgroup $Q$ if and only if $Q$ possesses an automorphism of order $p$ that acts irreducibly on $Q / \Phi(G)$. For any distinct $p$ and $q$, there exists a $\mathrm{B}(p, q)$-group. For $p>2, n \in \mathbb{N}$, the dihedral group of order $2 p^{n}$ is a $\mathrm{B}(2, p)$-group.

In this and the following paragraph we shall define some characteristic subgroups of an arbitrary group. Let $\mathfrak{B}_{q}(G)$ be the subgroup generated by normal Sylow $q$ subgroups (= derived subgroups) of all $\mathrm{B}(p, q)$-subgroups in $G(p \in \pi(G)-\{q\})$. By the Frobenius Normal $p$-Complement Theorem, $\mathfrak{B}_{q}(G)=\{1\}$ if and only if $G$ is $q$-nilpotent. Set $\mathfrak{B}(G)=\prod_{q \in \pi(G)} \mathfrak{B}_{q}(G)$.

Let $\Theta_{1}(G)$ denote the subgroup generated by all $\Theta_{1}$-subgroups of $G ; \Theta_{1}(G)=$ $\{1\}$ if and only if $G$ is a $\Theta$-group. $\Theta_{1}(G)$ is characteristic in $G$. We shall show (see the lemma below) that $G / \Theta_{1}(G)$ is a $\Theta$-group for some $\Theta$ 's.

Let $G=A \Theta_{1}(G)$ be such that the subgroup $A$ is as small as possible. Then $\Theta_{1}(A) \leq A \cap \Theta_{1}(G) \leq \Phi(A)$ is nilpotent. Hence, if $G / \Theta_{1}(G)$ is not a $\Theta$-group, there exists in $G$ a non- $\Theta$-subgroup $A$ such that $\Theta_{1}(A) \leq \Phi(A)$; in particular, all $\Theta_{1}$-subgroups of $A$ are nilpotent. Let, in addition, $\Theta=$ nilpotency. It follows from the remark above that then $A$ is nilpotent so $G / \Theta_{1}(G)$ is nilpotent. We generalize this observation in the following

Lemma. Suppose that all $\Theta_{1}$-subgroups of a group $G$ are not nilpotent. Then $G$ has a $\Theta$-subgroup $A$ such that $G=A \Theta_{1}(G)$. In particular, $G / \Theta_{1}(G)$ is a $\Theta$-group.

Proof. Suppose that the lemma has been proved for all proper subgroups of $G$. We may assume that $\Theta_{1}(G)>\{1\}$ (otherwise, $G$ is a $\Theta$-group, and the lemma is obvious). Let $A$ be a minimal subgroup of $G$ such that $G=A \Theta_{1}(G)$. Since $\Theta_{1}(G)$ is nonnilpotent, it is not contained in $\Phi(G)$. It follows that $A<G$. By the induction hypothesis, $A / \Theta_{1}(A)$ is a $\Theta$-group. Since $\Theta_{1}(A) \leq A \cap \Theta_{1}(G) \leq \Phi(A)$, 
we get $\Theta_{1}(A)=\{1\}$ since all $\Theta_{1}$-subgroups of $G$ are not nilpotent. This means that $A$ is a $\Theta$-group so $G / \Theta_{1}(G) \cong A / A \cap \Theta_{1}(G)$ is also a $\Theta$-group, as desired.

In particular, the lemma is true for $\Theta$ such that all nilpotent groups are $\Theta$ groups, but the assumption in the lemma is weaker. In fact, consider the case when $\Theta=$ commutativity and $G$ is a nonabelian group all of whose Sylow subgroups are abelian. Then all $\Theta_{1}$-subgroups of $G$ are nonnilpotent (however, there are nilpotent $\Theta_{1}$-groups). By the lemma, $G / \Theta_{1}(G)$ is abelian.

It is easy to show that the lemma is not true for $\Theta$ 's such that some $\Theta_{1}$-groups are nilpotent. In fact, if $\Theta$ is such that only the identity group is a $\Theta$-group, $\Theta_{1}(G)$ is the subgroup of $G$ generated by the elements of prime orders; in that case, the structure of $G / \Theta_{1}(G)$ may be very complicated.

Remark 1. Suppose, in addition, that a tower of $\Theta$-groups is a $\Theta$-group and all subgroups of prime orders are $\Theta$-groups (in that case, we will call $\Theta$ strong). We will prove that if $G$ is a $\Theta_{1}$-group, then $G / \Phi(G)$ is a nonabelian simple group. Indeed, let $N$ be a maximal normal subgroup of $G$ and $H$ a maximal subgroup of $G$. Since $H N$ is a $\Theta$-group, we get $N \leq H$. It follows that $N=\Phi(G)$ and $G / \Phi(G)$ is simple. It remains to show that $G / \Phi(G)$ is nonabelian. Assume that this is not true. Then $|G: \Phi(G)|=p$, a prime, so $\Phi(G)$ is maximal in $G$. It follows that $\Phi(G)$ is a unique maximal subgroup of $G$ so $G$ is a cyclic $p$-group. By definition, $|G|>p$ and $\Phi(G)$ is a $\Theta$-group. It follows that $G$, as a tower of $\Theta$-groups, is a $\Theta$ group, which is a contradiction. Note that solvability and $\pi$-separability are strong properties.

Remark 2. For strong properties $\Theta$, one can say more than the lemma says. Indeed, in the lemma, let $\Theta$ be a strong property and let $G$ not be a $\Theta$-group. Then $G / \Theta_{1}(G)$ is a $\Theta$-group, by the lemma. We claim that, in fact, $G / K$ is not a $\Theta$ group if a normal subgroup $K$ of $G$ is properly contained in $\Theta_{1}(G)$. Assume that this is not true. Then $G$ has a $\Theta_{1}$-subgroup $L$ such that $L \not \leq K$. Since $L / L \cap K$ and $K \cap L$ are $\Theta$-groups so is $L$, which is not the case. In particular, if $\Theta=$ solvability, then $\Theta_{1}(G)$ is the last member of the derived series of $G$.

Let $\mathfrak{S}(G)$ be the subgroup generated by all minimal nonnilpotent subgroups of $G$. By the lemma, $\mathrm{H}(G) \leq \mathfrak{S}(G)$. It follows that if all minimal nonnilpotent subgroups are normal in $G$, then $G / \mathrm{F}(G)$ is an extension of a direct product of elementary abelian groups by a nilpotent group. Indeed, if $K$ is generated by normal maximal subgroups of all minimal nonnilpotent subgroups of $G$, then $K \leq \mathrm{F}(G)$ and $\mathfrak{S}(G) / K$ is generated by normal subgroups of prime orders. But we do not know whether the nilpotent length of $G$ is bounded if all its minimal nonabelian subgroups are normal. In general, the inequality $\mathrm{H}(G) \neq \mathfrak{S}(G)$ is possible. In fact, let $G=\mathrm{S}_{n}$, $n>3$. Then it is easy to check that $\mathfrak{S}(G)=G$ : the general case follows from the case $n=4$, for which our assertion is trivial. Since $\mathrm{H}(G)=\mathrm{A}_{n}$, our claim follows.

Let $\pi$ be a set of primes. A group $G$ is said to be $\pi$-decomposable if a $\pi$-Hall subgroup is a direct factor of $G$. Let $K$ be the subgroup generated by all minimal nonnilpotent subgroups of $G$ of orders divisible by a fixed prime $p$. We claim that $G / K$ is $p$-decomposable. Indeed, let $\Theta=p$-decomposability. By [Hup], Satz 4.5.4, and basic properties of $p$-solvable groups, $\Theta_{1}$-groups are minimal nonnilpotent of orders divisible by $p$. Therefore, $K=\Theta_{1}(G)$. By the lemma, $G / K$ is a $\Theta$-group (i.e., it is $p$-decomposable), as claimed. Similarly, if $K$ is generated by all minimal 
non- $\pi$-decomposable subgroups of $G$, then, by the lemma, $G / K$ is $\pi$-decomposable. (Note that the minimal non- $\pi$-decomposable groups are not classified.)

Our principal result is the following

Theorem. (a) $\mathfrak{B}_{q}(G)=O^{q, q^{\prime}}(G)$, i.e., commutator subgroups of all $B(p, q)$ subgroups of $G(p \in \pi(G)-\{q\})$ generate $O^{q, q^{\prime}}(G)$.

(b) $\mathfrak{B}(G)=H(G)$. In other words, the subgroup, generated by commutator subgroups of all $B(p, q)$-subgroups of $G$, where $p, q$ run over the set $\pi(G)$, coincides with the last member of the lower central series of $G$.

Proof. (a) Assume that $\mathfrak{B}_{q}(G) \not \leq \mathrm{O}^{q, q^{\prime}}(G)$. Then $G$ has a $\mathrm{B}(p, q)$-subgroup $F=$ $P \cdot Q$, where $p \in \pi(G)-\{q\}, P \in \operatorname{Syl}_{p}(F)$ and $F^{\prime}=Q \in \operatorname{Syl}_{q}(F)$ such that $Q \not \leq \mathrm{O}^{q, q^{\prime}}(G)$. Then $\bar{F}=F / F \cap \mathrm{O}^{q, q^{\prime}}(G)\left(\cong F \mathrm{O}^{q, q^{\prime}}(G) / \mathrm{O}^{q, q^{\prime}}(G)\right)$ is of order divisible by $q$; therefore, $\bar{F}$ is not $q$-nilpotent (otherwise, $F$ has a normal subgroup of index $q$ which is not the case since $\left|F: F^{\prime}\right|$ is a power of $\left.p \neq q\right)$. Since every epimorphic image of $F$ is nilpotent or a $\mathrm{B}(p, q)$-group, it follows that $\bar{F}$ is a $\mathrm{B}(p, q)$ group. Thus, a non- $q$-nilpotent group $\bar{F}$ is isomorphic to a subgroup of the $q$ nilpotent group $G / \mathrm{O}^{q, q^{\prime}}(G)$, which is a contradiction. Hence $\mathfrak{B}_{q}(G) \leq \mathrm{O}^{q, q^{\prime}}(G)$. Recall that $\mathrm{O}^{q, q^{\prime}}(G)$ is contained in every normal subgroup $N$ of $G$ such that $G / N$ is $q$-nilpotent. Therefore, to prove the reverse inclusion, it is enough to show that $G / \mathfrak{B}_{q}(G)$ is $q$-nilpotent. Assume that $G / \mathfrak{B}_{q}(G)$ is not $q$-nilpotent. Then it has an $\mathrm{S}(p, q)$-subgroup $\bar{S}=S / \mathfrak{B}_{q}(G)$, by the Frobenius Normal $q$-Complement Theorem. Let $A$ be a smallest subgroup such that $S=A \mathfrak{B}_{q}(G)$. Then $A \cap \mathfrak{B}_{q}(G) \leq$ $\Phi(A)$ and $A / A \cap \mathfrak{B}_{q}(G)$ is an $\mathrm{S}(p, q)$-group since it is isomorphic to $\bar{S}$. It follows that $A / \Phi(A)$ is an $\mathrm{S}(p, q)$-group as a nonnilpotent epimorphic image of the $\mathrm{S}(p, q)$ group $\bar{S}$. Therefore, by Definition $1, A$ is a $\mathrm{B}(p, q)$-subgroup of $G$. Since $q$ divides $|\bar{S}|=\left|A \mathfrak{B}_{q}(G) / \mathfrak{B}_{q}(G)\right|$, the Sylow $q$-subgroup of $A$ is not contained in $\mathfrak{B}_{q}(G)$, contrary to the definition of the last subgroup. Thus, $G / \mathfrak{B}_{q}(G)$ is $q$-nilpotent so $\mathrm{O}^{q, q^{\prime}}(G) \leq \mathfrak{B}_{q}(G)$, and (a) follows.

(b) Let us prove that $K=\prod_{q \in \pi(G)} \mathrm{O}^{q, q^{\prime}}(G)$ is equal to $\mathrm{H}(G)$. Since $G / K$ is $q$-nilpotent for all $q \in \pi(G)$, by (a), it is nilpotent, and so $\mathrm{H}(G) \leq K$. The reverse inclusion is evident since $\mathrm{O}^{q, q^{\prime}}(G) \leq \mathrm{H}(G)$ for all $q \in \pi(G)$. Therefore, $K=\mathrm{H}(G)$. By (a),

$$
\mathfrak{B}(G)=\prod_{p \in \pi(G)} \mathfrak{B}_{p}(G)=\prod_{p \in \pi(G)} \mathrm{O}^{p, p^{\prime}}(G)=K=\mathrm{H}(G),
$$

completing the proof of (b).

If $K$ is the subgroup generated by normal Sylow subgroups of all minimal nonnilpotent subgroups of $G$, then $G / K$ is not necessarily nilpotent (let $G$ be the dihedral group of order $2 p^{n}, p>2, n>1$ ). Moreover, we cannot prove that, in the case under consideration, $G / K$ is solvable.

In the following paragraph we will use the Baer-Suzuki Theorem (see [HB], Theorem 9.7.8):

$\left.{ }^{*}\right)$ If $x$ is a $p$-element of $G$, then $x \in \mathrm{O}_{p}(G)$ if and only if $\left\langle x, x^{y}\right\rangle$ is a $p$-subgroup for all $y \in G$.

The following result is a consequence of $(*)$ (see [B], p. 27, where another proof is given):

(**) If $G$ has no $\mathrm{S}(2, p)$-subgroups for all odd $p \in \pi(G)$, it is 2-closed. 
Let us prove that if $\Theta=$ commutativity (or cyclicity), then $G / \Theta_{1}(G)$ is 2-closed. Let $G$ be a counterexample of minimal order. Suppose that $\Theta_{1}(G)$ is not nilpotent. Then $\Theta_{1}(G)$ has a nonnormal Sylow subgroup $P$. By Frattini's Lemma, $G=$ $\Theta_{1}(G) \mathrm{N}_{G}(P)$. Since $\Theta_{1}\left(\mathrm{~N}_{G}(P)\right) \leq \Theta_{1}(G) \cap \mathrm{N}_{G}(P)$ and $\mathrm{N}_{G}(P)<G$, it follows by the induction hypothesis that $\mathrm{N}_{G}(P) / \Theta_{1}\left(\mathrm{~N}_{G}(P)\right.$ ) (and so its epimorphic image $\left.\mathrm{N}_{G}(P) / \mathrm{N}_{G}(P) \cap \Theta_{1}(G)\right)$ is 2-closed. Since $G / \Theta_{1}(G)$ is isomorphic to the last group, it is also 2-closed. Thus, $\Theta_{1}(G)$ is nilpotent so all $\Theta_{1}$-subgroups of $G$ are nilpotent. Since $\mathrm{S}(2, p)$-groups, $p>2$, are $\Theta_{1}$-groups (see [Hup], Satz 3.5.2), $G$ has no $\mathrm{S}(2, p)$ subgroups for all odd $p \in \pi(G)$. It follows from (**) then that $G$ is 2-closed, as desired.

If, as in the previous paragraph, $\Theta=$ commutativity, then all nonabelian Sylow subgroups are contained in $\Theta_{1}(G)$ (see added in proof).

In view of what has been said, it is interesting to study the groups whose minimal nonabelian subgroups are all nilpotent (such groups are 2 -closed, by $(* *)$ ).

I am indebted to R. Solomon and the referee for useful comments and suggestions.

\section{ADDED IN PROOF}

It is easy to deduce from this that $G / K$, where $K=\Theta_{1}(G)$, is abelian. Indeed, assume that $G / K$ is nonabelian. Let $S / K$ be a minimal nonabelian subgroup of $G / K$; then $S / K$ is nonnilpotent. Let $A$ be a minimal subgroup such that $S=A K$; then $A$ is a $B(p, q)$-subgroup. Since Sylow subgroups of $A$ are abelian, $Z(A)$ is a $p$-subgroup. It follows that $A=\Theta_{1}(A) \leq K$, a contradiction.

\section{REFERENCES}

[B] Y. Berkovich, A theorem on nonnilpotent solvable subgroups of finite groups, in 'Finite groups', Nauka i Tehnika, Minsk, 1966, pp. 24-39 (Russian).

[Gas] W. Gaschütz, Über die $\Phi$-Untergruppe endlichen Gruppen, Math. Z. 58 (1953), 160-170. MR 15:285c

[Gol] Yu.A. Golfand, On groups all of whose subgroups are nilpotent, Dokl. Akad. Nauk SSSR 60 (1948), 1313-1315 (Russian).

[H] M. Hall, The theory of groups, Macmillan, New York, 1959. MR 21:1996

[Hup] B. Huppert, Endliche Gruppen, Bd. 1, Springer, Berlin, 1967.

[HB] B. Huppert and N. Blackburn, Finite Groups II, Springer-Verlag, Berlin, 1982. MR 84i:20001a

[I] N. Ito, Note on (LM)-groups of finite order, Kodai Math. Seminar Report (1951), 1-6. MR 13:317a

[R] L. Redei, Die endlichen einstufig nichtnilpotenten Gruppen, Publ. Math. Debrecen 4 (1956), 303-324.

[S] O.Yu. Schmidt, Groups all of whose subgroups are nilpotent, Mat. Sb. 31 (1924), 366-372 (Russian).

Department of Mathematics, University of Haifa, Mount Carmel, Haifa 31905, Israel

E-mail address: berkov@mathcs2.haifa.ac.il 